\title{
Proceeding Paper \\ Characterization and Comparison of the Relative Humidity Response of Hydromorphic Polymers in Long-Period Fiber Grating Structures ${ }^{\dagger}$
}

\author{
Bernardo Dias $1,2, *\left(\mathbb{0}\right.$, João P. Mendes ${ }^{1,2,3}\left[\right.$, José M. M. M. de Almeida ${ }^{1,4}$ (1) and Luís C. C. Coelho ${ }^{1,2, *(1)}$ \\ 1 INESC TEC-Institute for Systems and Computer Engineering, Technology and Science, Faculty of Sciences, \\ University of Porto, 4169-007 Porto, Portugal; joao.p.mendes@inesctec.pt (J.P.M.); \\ jmmma@utad.pt (J.M.M.M.d.A.) \\ 2 Department of Physics and Astronomy, Faculty of Sciences, University of Porto, 4169-007 Porto, Portugal \\ 3 Chemistry Research Unit, Faculty of Sciences, University of Porto, 4169-007 Porto, Portugal \\ 4 Department of Physics, School of Science and Technology, University of Trás-os-Montes e Alto Douro, \\ 5001-801 Vila Real, Portugal \\ * Correspondence: bernardo.s.dias@inesctec.pt (B.D.); luis.c.coelho@inesctec.pt (L.C.C.C.) \\ + Presented at the 1st International Electronic Conference on Chemical Sensors and Analytical Chemistry, \\ 1-15 July 2021; Available online: https:/ / csac2021.sciforum.net/.
}

check for updates

Citation: Dias, B.; Mendes, J.P.; de Almeida, J.M.M.M.; Coelho, L.C.C. Characterization and Comparison of the Relative Humidity Response of Hydromorphic Polymers in Long-Period Fiber Grating Structures. Chem. Proc. 2021, 5, 42. https:// doi.org/10.3390/CSAC2021-10461

Academic Editor:

Nicole Jaffrezic-Renault

Published: 30 June 2021

Publisher's Note: MDPI stays neutral with regard to jurisdictional claims in published maps and institutional affiliations.

Copyright: (C) 2021 by the authors. Licensee MDPI, Basel, Switzerland. This article is an open access article distributed under the terms and conditions of the Creative Commons Attribution (CC BY) license (https:// creativecommons.org/licenses/by/ $4.0 /)$.

\begin{abstract}
Relative humidity monitorization is of extreme importance on scientific and industrial applications, and fiber optics-based sensors may provide solutions where other types of sensors have limitations. In this work, fiber optics' sensors were fabricated by combining Long-Period Fiber Gratings with three different humidity-responding polymers, namely Poly(vinyl alcohol), Poly(ethylene glycol) and Hydromed ${ }^{\mathrm{TM}}$ D4. The performance of the multiple sensors was experimentally tested and crossed with numerical simulations, which provide a comparison with the expected response given the optical properties of the materials.
\end{abstract}

Keywords: relative humidity sensors; long-period fiber gratings; hydromorphic polymers; optical sensors

\section{Introduction}

The real time monitoring of relative humidity on scientific and industrial applications is of extreme importance and many types of sensors have been developed, mostly based on capacitive or resistive structures which may display some flaws, such as not being immune to electromagnetic radiation, and not fit to extreme and harsh environments (such as underwater applications). The usage of hydromorphic polymers in optical fiber structures is a thoroughly explored field of research with many publications associated [1-4], some of which include the usage of Poly(vinyl alcohol) (PVA), Poly(ethylene glycol) (PEG) or the combination of the two [1]. The polymers display a refractive index (RI) that decreases with the absorption of water molecules, also displaying considerable swelling effects. These changes can be tracked by analyzing the spectral characteristics of specific optical structures such as long-period fiber gratings (LPFG) $[5,6]$. LPFGs consist of a periodic modulation to the RI of the core of the fiber, resulting in coupling of light from the fundamental core mode to the co-propagating cladding modes, which creates rejection bands in the transmission spectrum at specific wavelengths with high sensitivity to the surrounding RI. By coating LPFGs with the aforementioned materials, this refractometer can be used to measure relative humidity.

Preliminary results regarding LPFGs fabricated in single mode optical fibers and coated with three different humidity-responding polymers PVA, PEG and a Hydrogel (Hydromed ${ }^{\mathrm{TM}}$ D4) are presented. In the case of Hydrogel, no such work seems to have been published previously. When placed in an environment with varying humidity, the polymers 
used as coating of the LPFG will both swell and change their RI, resulting in changes in the coupling conditions of the light to the cladding modes. Besides the experimental work, in order to have a better understanding of the effect of the coatings in the LPFG, simulations based on coupled-mode theory [7] were implemented, which allow understanding of the range of action of the LPFGs and optimization of their performance.

\section{Materials and Methods}

In order to test the capabilities of the polymers, several solutions of different concentrations were made, and thin layers were deposited around LPFGs previously fabricated in single mode fibers (Corning SMF28e) with the electric arc technique following the procedure described in [6]. This allowed the calibration of the wavelength shift and optical power shift induced by the coating process, meaning that for a given LPFG it is possible to choose the concentration of the coating solution that gives both visibility of the rejection band and high sensitivity to the $\mathrm{RH}$ variation. At the same time, given that the $\mathrm{RH}$ response of the sensor is a result of changes in RI and the thickness of the coating layer, testing LPFGs coated with solutions of different concentrations will also allow exploration of different responses caused by this variation of parameters.

\subsection{Polymers Used for Humidity Sensing}

Three different polymers were chosen and compared, a hydrogel (HydroMed ${ }^{\mathrm{TM}}$ D4), PVA (polyvinyl alchohol) and PEG (polyethylene glycol).

PVA is a water-soluble polymer, which has been studied extensively in the past as a coating for fiber optics-based humidity sensors [1-4]. It is a hydrophilic polymer that absorbs and desorbs water, with variations in the RI between 1.49 to 1.45 (at $1310 \mathrm{~nm}$ ) according to [2] or between 1.49 to 1.34 (at $1550 \mathrm{~nm}$ ) according to [4].

PEG is a polymer derived from petroleum containing ether linkages in its main chain, thus being referred to as a polyether. While there are some studies using this polymer for humidity sensing [8], only one application of this polymer to LPFG was found [1], which relied on a combination of PVA and PEG. The RI of PEG is lower than that of PVA, varying from 1.455 to $1.413\left(\mathrm{n}_{\mathrm{D}}\right)$ [8].

HydroMed $^{\mathrm{TM}} \mathrm{D}$ is a series of ether-based hydrophilic urethanes fabricated by AdvanSource Biomaterials [9]. These materials were designed to respond by expanding when in contact with water molecules, with each product having different responses to different water contents. The polymer is distributed in granular form, which can be dissolved in many different solvents, of which ethanol was chosen.

\subsection{Fabrication of the Coating Solutions and Coating Process}

The solutions required different procedures in order to be dissolved. Generally, a solvent with low boiling point such as ethanol is preferred because it will lead to a faster evaporation and consequently the coating process will be faster. Nevertheless, given the low solubilities of PVA and PEG in ethanol, deionized water was used instead, which requires a longer time to fully evaporate and thus deposit the coating. In the case of hydrogel, the solvent was ethanol. Solutions with three different concentrations were produced, namely 10, 7.5 and 5\% wt/wt, prepared by dissolving the high purity granules with the solvent, in the corresponding ratio. The PVA solution was obtained by adding PVA to water in a concentration of $10 \% w t / w t$, and stirring for $3 \mathrm{~h}$ at $60{ }^{\circ} \mathrm{C}$. The same procedure was applied to obtain the 5 and $7.5 \%$ concentration solutions and in the case of the PEG Scheme 50, 75 and 100\% wt/wt.

The fabricated LPFGs were coated with all the polymers by stretching and dipping horizontally with a small angle between the longitudinal axes of the fiber and a V groove filled with the polymer. The fiber was then left at room temperature to ensure solvent evaporation.

This coating process was done for each sensor, thus justifying the need for different concentrations of the solutions, which have different viscosities and thus will create thicker 
coatings the more concentrated the solution is. In addition, calibration of the appropriate concentration of the solution to a given LPFG, in order to ensure that the wavelength and optical power shifts do not render the LPFG outside certain desirable parameters (such as minimum wavelength in the interval 1500 to $1600 \mathrm{~nm}$ ), was performed.

\subsection{Humidity Measurements}

In order to calibrate the fabricated sensors, an experimental setup was devised in which the environment's humidity could be controlled and measured (Figure 1).

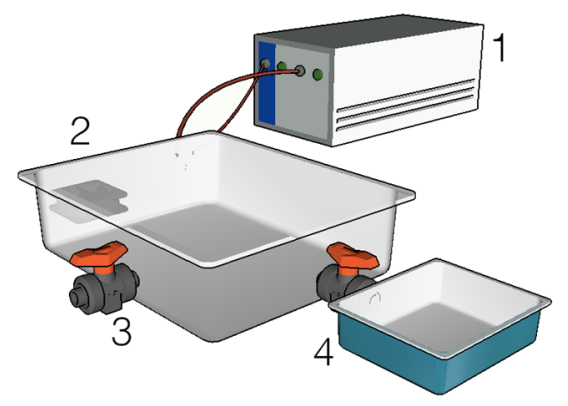

Figure 1. Experimental setup created to measure changes in LPFG spectra in varying values of relative humidity. 1-Interrogation unit; 2-Humidity Chamber; 3-Exterior valve; 4-Humidity valve.

This chamber was made from a container with two valves that connected to the exterior (where the RH was around 50\%), and a side container with water connected by a valve to the main one. A small fan was also placed inside the container in order to promote faster diffusion of the water molecules in the air. This setup allowed the variation of internal humidity in varying rates, depending on the fan speed.

The humidity chamber allowed for the insertion of two LPFGs at once, one with the humidity sensor and another for thermal compensation. The two fibers were placed in a stand with weights in their extremities, guaranteeing that the sensor was fully stretched at all times. The fiber was connected to an interrogation unit (HBM Fibersensing FS22 Braggmeter) on the outside, which recorded the spectra at all times. Also inside the container was a humidity and temperature sensor (DHT22), which has a typical accuracy of $\pm 2 \% \mathrm{RH}$ and $\pm 0.5^{\circ} \mathrm{C}$ and a working range of $0 \% \mathrm{RH}$ to $100 \% \mathrm{RH}$ and $-40{ }^{\circ} \mathrm{C}$ to $80{ }^{\circ} \mathrm{C}$. This sensor was connected to an Arduino Uno unit which displayed the humidity and temperature values every $12 \mathrm{~s}$, allowing to record every LPFG sensor spectra along with the respective humidity and temperature value.

\subsection{Experimental Procedure}

In order to provide a preliminary characterization of the sensors, the LPFG spectra were taken in descending RH values. Firstly, the valve to the side container with water was opened and the fan was turned on for $15 \mathrm{~min}$. This ensured that the chamber attained the maximum possible values of humidity, which was recorded at $99 \% \mathrm{RH}$. After this process, the fan was turned off and after a minute of stabilization, the valve to the external environment was opened, and the measurements initiated. Due to the opened valve, the relative humidity decreased by $1 \%$ every 2 to $3 \mathrm{~min}$, which provided an ideal stable decrease in humidity for measurements. The spectra were recorded for every decrease in $1 \%$ RH. The data were plotted in real time to determine the spectral evolution and the working range of the fabricated sensor.

\section{Results}

\subsection{Humidity Measurements}

As described in the experimental procedure, for each fabricated sensor its response to $\mathrm{RH}$ variations was tested in its working range for both descending and ascending variations. In this section, the results obtained by direct comparison from LPFGs coated 
with the same polymers at different concentrations are summarized, in order to choose one that has an optimal performance.

\subsubsection{PVA Coated LPFGs}

Figure 2 shows the results obtained from LPFGs coated with thin films of PVA from solutions with different concentrations.

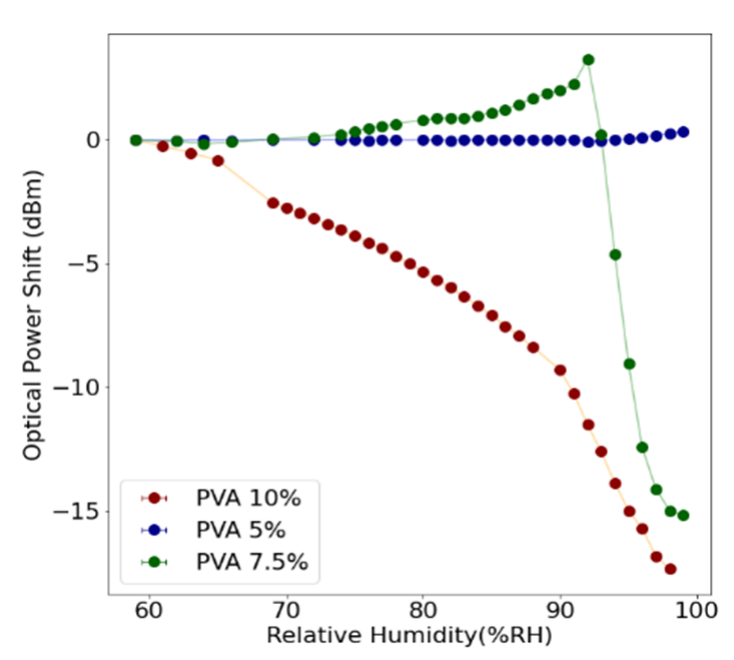

(a)

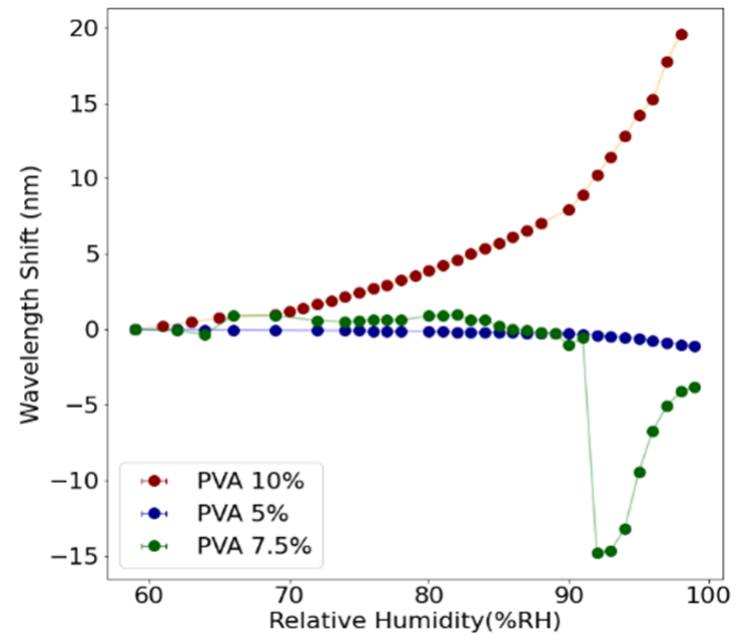

(b)

Figure 2. Variation of the spectra of the three PVA coated LPFG sensors and their measured response: (a) Optical Power Shift response; (b) Wavelength Shift Response.

The sensor coated with a $5 \%$ wt /wt solution displays very low response to $\mathrm{RH}$ variations, meaning that the obtained layer is too thin and making the sensor not suitable. On the other hand, the 7.5 and $10 \%$ sensors display very different behaviors, with the $10 \%$ $w t / w t$ one displaying the typical LPFG wavelength shift curve due to the external RI being near the cladding index $\left(\mathrm{n}_{\text {clad }}=1.44\right)$, but the $7.5 \%$ sensor displays a transition characteristic from guided to leaky modes, meaning that in this case the external RI transitioned from $\mathrm{n}<\mathrm{n}_{\text {clad }}$ to $\mathrm{n}>\mathrm{n}_{\text {clad, }}$, which disagrees with the RI values reported in [2] (in which the PVA's $\mathrm{RI}$ as a value of 1.45 at $100 \% \mathrm{RH}$ ). The discontinuity seen in Figure $2 \mathrm{~b}$ shows that at $91 \% \mathrm{RH}$ the layer of PVA matches the cladding index, meaning that the fiber is suddenly thicker, and the mode has a discontinuous transition. This process also renders the $7.5 \%$ sensor not suitable. On the other hand, the $10 \% w t / w t$ sensor shows very good response with high sensitivity especially in the region above $90 \% \mathrm{RH}$, but overall good performance in the tested range.

Considering the response of the LPFG coated with 10\% wt/wt PVA solution, a comparison with the 3-layer coupled-mode theory simulations of LPFGs [7] was established, in order to check if the response of the sensor matches the values of [2] or [4]. Good agreement is seen between the simulations considering the RI variations of 1.345 to 1.43 reported in [4] and the experimental data (Figure 3), showing also that in this case the coating layer can be approximated to an infinite medium, because the penetration depth of the evanescent field of the cladding mode is inferior to the PVA coating thickness. The simulations show that the $10 \% w t / w t$ is working on the high sensitivity zone of the LPFG, justifying the excellent performance of the sensor seen in Figure 2. Given the excellent performance of this sensor, the RH cycle was repeated for increasing RH values and a second LPFG was fabricated, in order to test the reproducibility of this structure. The results shown in Figure 3 show that excellent reproducibility was observed. 


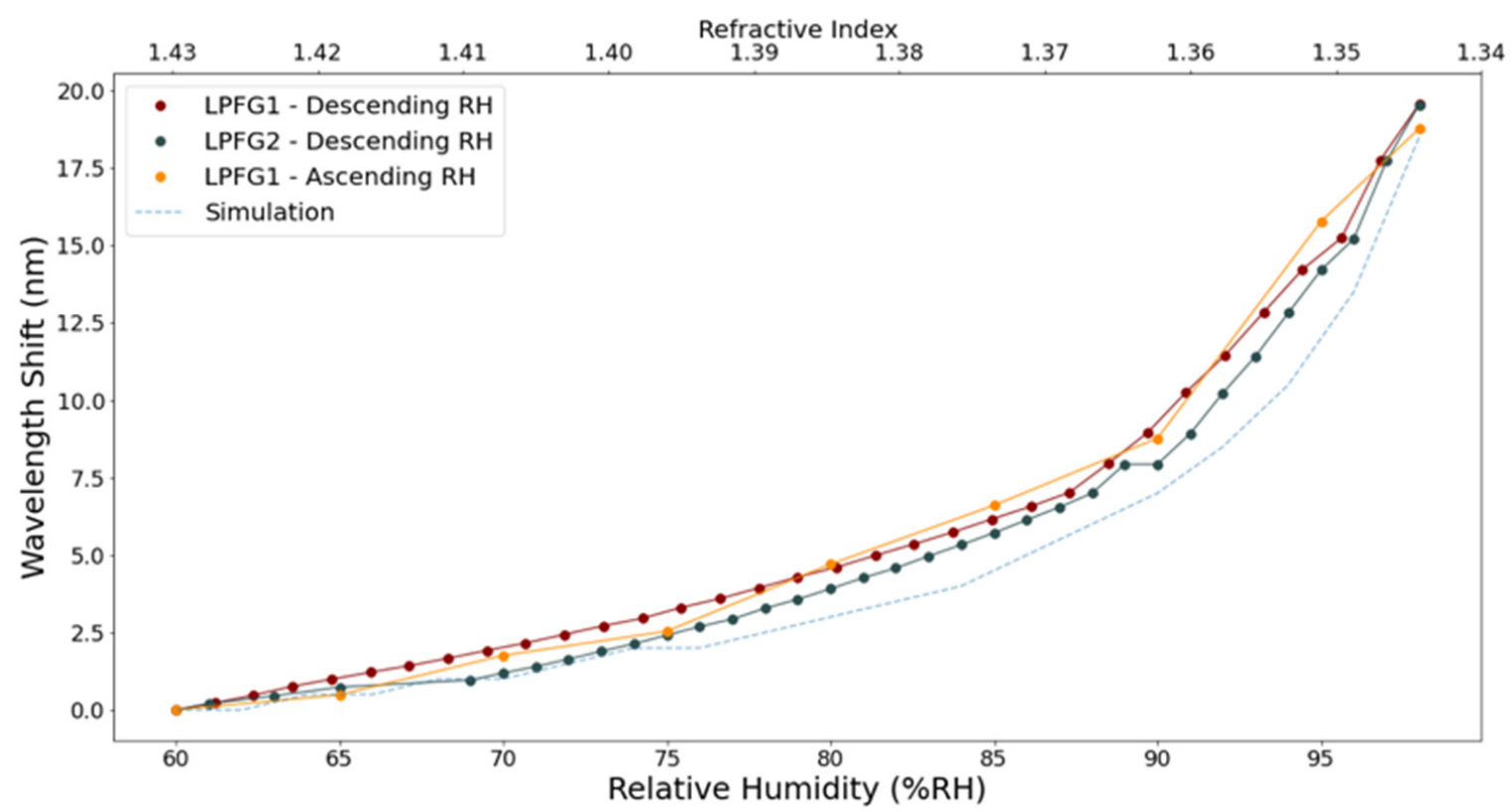

Figure 3. Comparison between the experimental wavelength shift of two $10 \%$ wt $/ w t$ PVA LPFG and the simulations, as function the external refractive index and relative humidity.

\subsubsection{PEG-Coated LPFGs}

Figure 4 shows the optical power and wavelength shifts in varying humidity for the cases of the PEG-coated LPFGs. In this case, the transition associated with the external medium having $\mathrm{n}>\mathrm{n}_{\text {clad }}$ is seen in all cases, and at lower RH values than PVA (80 to $87 \% R H)$, as can be seen in Figure $4 \mathrm{~b}$. This result was to be expected, given that the RI of PEG is lower than that of PVA, ranging from around 1.44 to 1.413 in the range of 60 to $99 \% \mathrm{RH}$ (in the Sodium line) [8], meaning that the transition will happen at lower $\mathrm{RH}$ values. This renders the sensors not suitable in wavelength shift response, even though their optical power shift response (Figure 3a) could be used, in the 80 to $95 \% \mathrm{RH}$ in the case of the $75 \%$ wt $/ w t$ coated LPFG.

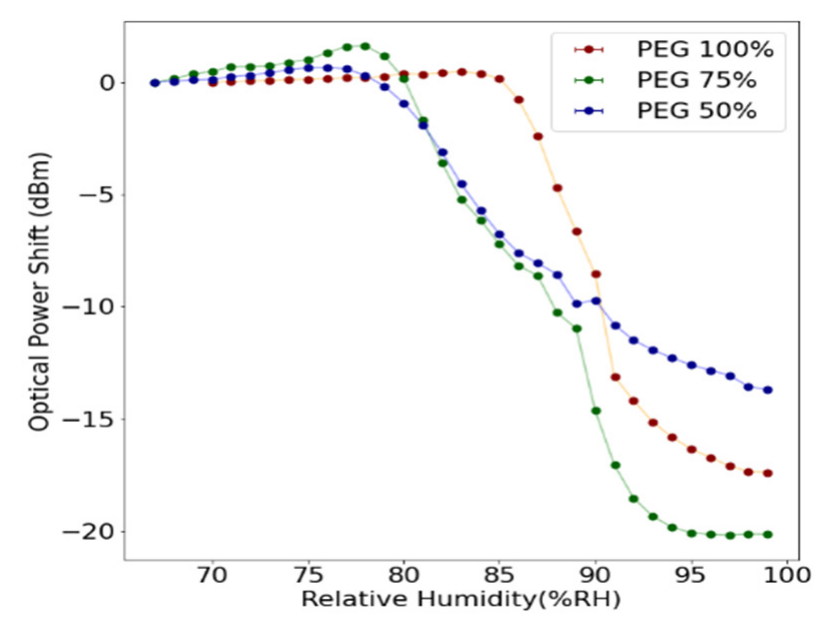

(a)

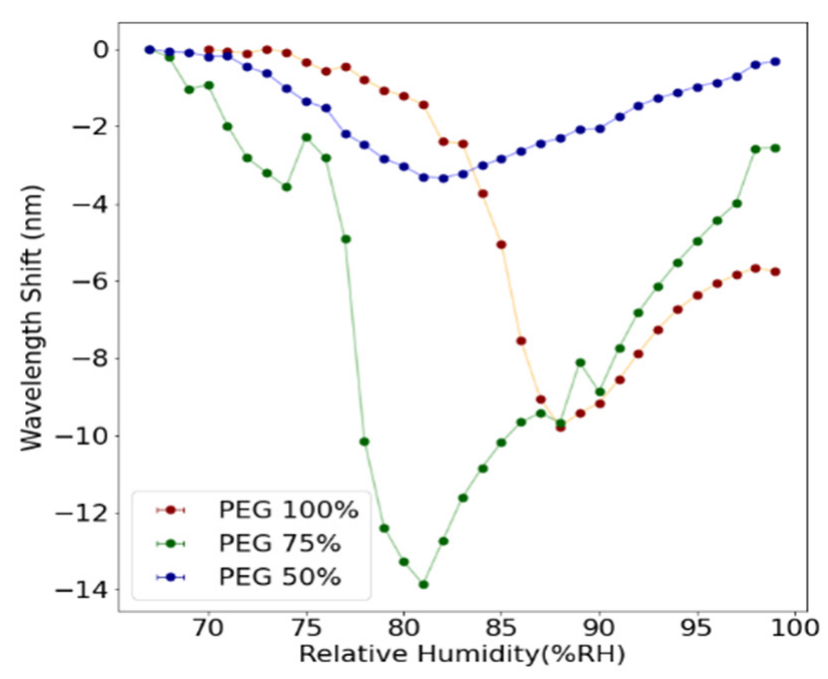

(b)

Figure 4. Variation of the spectra of the three PEG-coated LPFG sensors and their measured response: (a) Optical Power Shift response; (b) Wavelength Shift Response. 


\subsubsection{Hydrogel Coated LPFGs}

Figure 5 shows the optical power and wavelength shifts in varying humidity for the cases of the Hydrogel coated LPFGs. The responses in both wavelength and optical power shift seem similar to the case of the PEG coated LPFGs, with the transition of $\mathrm{n}>\mathrm{n}_{\text {clad }}$ to $\mathrm{n}<\mathrm{n}_{\text {clad }}$ happening in the range of $83 \%$ to $92 \% \mathrm{RH}$, which makes the wavelength shifts an unsuitable figure of merit for relative humidity monitoring. On the other hand, the optical power shift presents a suitable curve that enables the use of the structures as sensors, even though the sensitivity is considerably less than both the PVA and PEG cases.

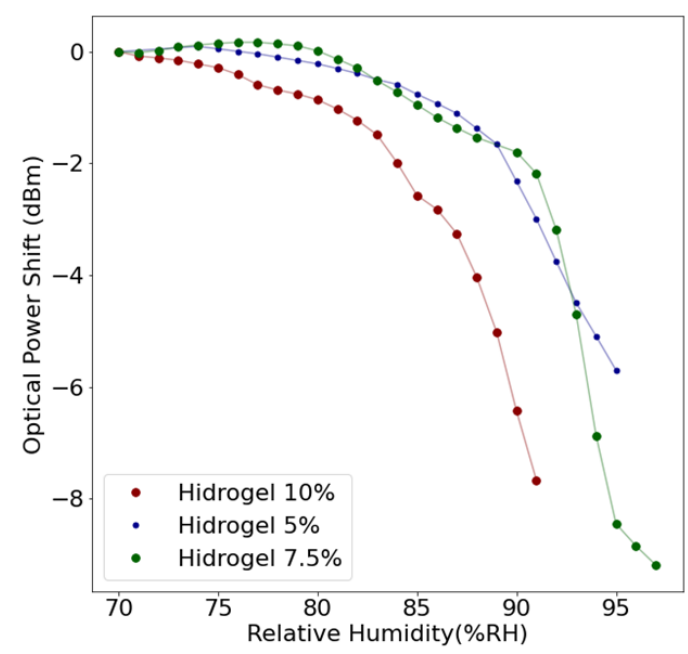

(a)

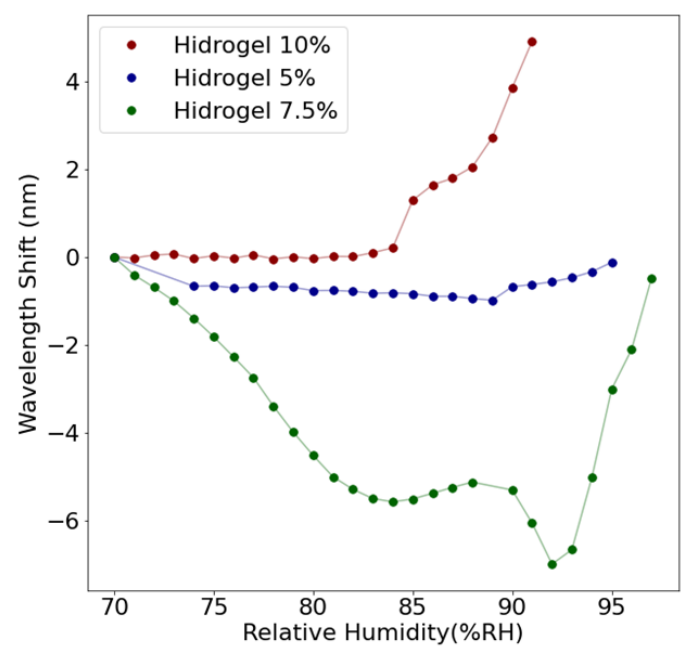

(b)

Figure 5. Variation of the spectra of the three Hydrogel coated LPFG sensors and their measured response: (a) Optical Power Shift response; (b) Wavelength Shift Response.

\section{Conclusions}

The response curves of LPFGs coated with three different humidity responding polymers (PVA, PEG and a Hydrogel) were obtained, with humidities varying from $60 \% \mathrm{RH}$ (or $70 \%$ RH, depending on the polymer) to $100 \%$ RH. Of all the fabricated sensors, the $10 \%$ $w t / w t$. PVA coated LPFG displays the best properties for relative humidity sensing, which were verified when comparing to numerical LPFG simulations.

Due to the variation of the optical properties of the polymers with varying humidity, namely the fact that the polymer's RI becomes larger than the cladding RI at low RH values, a transition from guided to leaky modes is seen, displaying a non-linear wavelength shift response, which renders it as an unsuitable figure of merit for $\mathrm{RH}$ sensing for most sensors. This conclusion shows that further work is needed to quantify the response of the three polymers to humidity variations, in order to optimize the sensors' performance and make them commercially viable. By fabricating new materials, which could possibly result from mixing the ones here studied, the desired optical properties (refractive index slightly below the cladding RI for all RH values and linear response) may be attained, creating a highly sensitive, viable solution for industrial and scientific application.

Supplementary Materials: The following are available online at https:/ /www.mdpi.com/article/10 $.3390 /$ CSAC2021-10461/s1.

Author Contributions: Conceptualization, B.D., J.M.M.M.d.A., L.C.C.C.; methodology, B.D., J.P.M., J.M.M.M.d.A., L.C.C.C.; formal analysis, B.D., J.M.M.M.d.A., L.C.C.C.; investigation, B.D., J.P.M., J.M.M.M.d.A., L.C.C.C.; writing-original draft preparation, B.D.; writing-review and editing, B.D., J.P.M., J.M.M.M.d.A., L.C.C.C.; supervision, J.M.M.M.d.A., L.C.C.C.; project administration, J.M.M.M.d.A., L.C.C.C. All authors have read and agreed to the published version of the manuscript. 
Funding: This work has received funding from the project "SolSensors-Development of Advanced Fiber Optic Sensors for Monitoring the Durability of Concrete Structures", with reference POCI-010145-FEDER-031220, supported by COMPETE 2020 and the Lisbon Regional Operational Program in its FEDER component, and by the budget of FCT Foundation for Science and Technology, I.P. Luis Coelho acknowledges the support from FCT research contract grant CEECIND/00471/2017.

Conflicts of Interest: The authors declare no conflict of interest. The funders had no role in the design of the study; in the collection, analyses, or interpretation of data; in the writing of the manuscript, or in the decision to publish the results.

\section{References}

1. Wang, Y.; Liu, Y.; Zou, F.; Jiang, C.; Mou, C.; Wang, T. Humidity Sensor Based on a Long-Period Fiber Grating Coated with Polymer Composite Film. Sensors 2019, 19, 2263. [CrossRef]

2. Gastón, A.; Pérez, F.; Sevilla, J. Optical Fiber Relative-Humidity Sensor with Polyvinyl Alcohol Film. Appl. Opt. 2004, 43, 4127-4132. [CrossRef]

3. Dong, X.; Li, T.; Liu, Y.; Li, Y.; Zhao, C.-L.; Chan, C.C. Polyvinyl Alcohol-Coated Hybrid Fiber Grating for Relative Humidity Sensing. J. Biomed. Opt. 2011, 16, 077001. [CrossRef]

4. Miao, Y.; Liu, B.; Zhang, H.; Li, Y.; Zhou, H.; Sun, H.; Zhang, W.; Zhao, Q. Relative Humidity Sensor Based on Tilted Fiber Bragg Grating With Polyvinyl Alcohol Coating. IEEE Photonics Technol. Lett. 2009, 21, 441-443. [CrossRef]

5. Vengsarkar, A.M.; Lemaire, P.J.; Judkins, J.B.; Bhatia, V.; Erdogan, T.; Sipe, J.E. Long-Period Fiber Gratings as Band-Rejection Filters. J. Lightwave Technol. 1996, 14, 58-65. [CrossRef]

6. Rego, G. Arc-Induced Long Period Fiber Gratings. J. Sens. 2016, 2016, e3598634. [CrossRef]

7. Erdogan, T. Cladding-Mode Resonances in Short- and Long-Period Fiber Grating Filters. J. Opt. Soc. Am. A JOSAA 1997, 14, 1760-1773. [CrossRef]

8. Acikgoz, S.; Bilen, B.; Demir, M.M.; Menceloglu, Y.Z.; Skarlatos, Y.; Aktas, G.; Inci, M.N. Use of Polyethylene Glycol Coatings for Optical Fibre Humidity Sensing. Opt. Rev. 2008, 15, 84. [CrossRef]

9. Hydromed ${ }^{\mathrm{TM}}$ Product Datasheet. Available online: http://www.advbiomaterials.com/products/hydrophilic/HydroMed.pdf (accessed on 1 June 2021). 